\title{
Restricted exchange microenvironments for cell culture
}

\author{
Jan H. Hoh ${ }^{1,2}$, Jeffrey L. Werbin ${ }^{1,3}$, and William F. Heinz ${ }^{1,4}$ \\ ${ }^{1}$ Department of Physiology, Johns Hopkins School of Medicine, Baltimore, MD, USA, ${ }^{2}$ Present Address: \\ 6644 Finley Place, Boulder, CO 80301, USA, ${ }^{3}$ Present Address: 312 Dexter Ave N, Seattle, WA \\ 98109, USA, ${ }^{4}$ Present Address: Optical Microscopy and Analysis Laboratory, Frederick National Laboratory \\ for Cancer Research, Leidos Biomedical Research Inc., Frederick, MD, USA.
}

BioTechniques 64:101-106 (March 2018) doi 10.4155/btn-2017-0110

Keywords: diffusive exchange; hypoxia chamber; metabolite gradients; 1-D spheroid; cellular microenvironment

Supplementary material for this article is available at www.BioTechniques.com/article/btn-2017-0110

Metabolite diffusion in tissues produces gradients and heterogeneous microenvironments that are not captured in standard 2D cell culture models. Here we describe restricted exchange environment chambers (REECs) in which diffusive gradients are formed and manipulated on length scales approximating those found in vivo. In REECs, cells are grown in 2D in an asymmetric chamber $(<50 \mu \mathrm{L})$ formed between a coverglass and a glass bottom cell culture dish separated by a thin $(\sim 100 \mu \mathrm{m})$ gasket. Diffusive metabolite exchange between the chamber and bulk media occurs through one or more openings micromachined into the coverglass. Cell-generated concentration gradients form radially in REECs with a single round opening $(\sim 200 \mu \mathrm{m}$ diameter). At steady state only cells within several hundred micrometers of the opening experience metabolite concentrations that permit survival which is analogous to diffusive exchange near a capillary in tissue. The chamber dimensions, the openings' shape, size, and number, and the cellular density and metabolic activity define the gradient structure. For example, two parallel slots above confluent cells produce the 1D equivalent of a spheroid. Using REECs, we found that fibroblasts align along the axis of diffusion while MDCK cells do not. MDCK cells do, however, exhibit significant morphological variations along the diffusive gradient.

Diffusive exchange between capillaries and the tissues they serve gives rise to concentration gradients of soluble metabolites within the tissue, creating variations in the microenvironment on the scale of single cells. Even neighboring cells can be exposed to appreciably different concentrations of these molecules, and the gradients play a fundamental role in tissue physiology. For example, Krogh demonstrated in 1919 that diffusive exchange is a primary determinant of capillary density in muscle (1). Diffusive exchange of metabolites also plays a central role in pathologies such as cancer (2-4) and stroke $(5,6)$. Special attention has been paid to gradients of oxygen, glucose, and $\mathrm{pH}$, but blood delivers many different molecules that are used by cells in tissues (e.g. fatty acids, amino acids, vitamins, etc.). The circulatory system likewise removes a significant number of metabolic byproducts that are produced by the cells (7), notably lactate and carbonate, but also other molecules such as glutamate and citrate. Despite the importance of these concentration gradients, there are limited tools for reproducing them in cell culture.

Approaches to capturing diffusive gradients in cell culture models can be broadly divided into two types, one in which concentration gradients of some small number of known molecules are formed by experimentally manipulating the molecules of interest, and another in which gradients of all relevant molecules are self-generated by cells. One of the simplest systems for producing a gradient of a specific molecule is the Zigmond chamber, in which two media reservoirs are connected by a thin, 1D chamber (8). If the concentration of a molecule is different in the two reservoirs, a gradient through the thin chamber will form by diffusion, which will typically remain stable for hours. More recently, a range of sophisticated microfluidic approaches for defining and controlling gradients during cell culture have been developed (9). These offer

\section{METHOD SUMMARY}

We developed restricted exchange environment chambers, low-volume ( $50 \mu \mathrm{L})$, asymmetric chambers formed between a micromachined glass coverslip and a glass bottom cell culture dish, separated by a thin ( 100 $\mu \mathrm{m})$ gasket, in which stable, 2D, cell-generated diffusive gradients of oxygen and metabolites mimic those found in tissue and spheroids. 
superior control over shape and temporal changes in individual molecular gradients, but for model systems to reproduce the situation in a tissue, they must account for all of the metabolites and their distributions over time in the relevant tissue. That level of understanding is not presently available for any tissue. Furthermore, even if those data were available, it would be technically challenging and expensive to reproduce the number of gradients that occurs in vivo. Thus, these methods are best suited for studying the effects of concentration gradients of specific molecules on cells in an otherwise uniform background.

The most widely used cell culture model to capture in vivo-like concentration gradients is spheroids (10-12). Many cell types under suitable conditions will form small spheroidal clusters of cells, which grow to diameters on the order of 300-400 $\mu \mathrm{m}$. By virtue of the spheroid morphology, radial concentration gradients of metabolites are selfgenerated by the metabolic activity of the cells in the spheroid. These gradients have been directly measured for a limited number of molecules such as oxygen, glucose and lactate, but the presumption is that gradients form for all molecules produced or consumed. Thus, the concentrations of oxygen and glucose fall as a function of the radial distance from the spheroid surface, while lactate and carbonate increase. There are also marked physiological differences in cells depending on their distance to the surface of the spheroid. For example, cells near the surface are more likely to be mitotic while interior cells are more likely to become quiescent and undergo apoptosis. Spheroids have been extensively studied

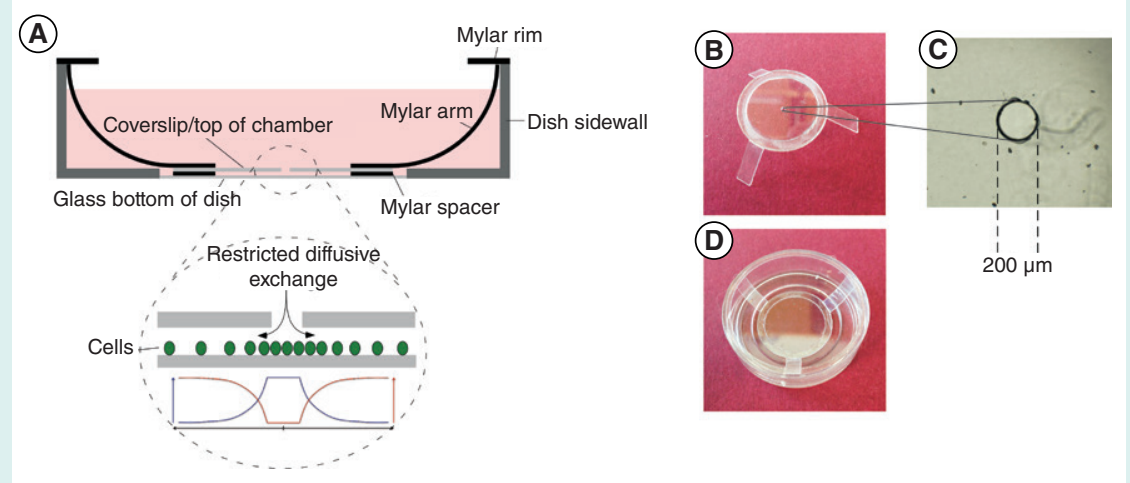

Figure 1. Design and fabrication of a REEC. (A) Schematic diagram showing a cross section of an assembled REEC without the dish cover. The illustration below shows how the opening in the chamber allows for limited diffusive exchange. The plot indicates the relationship between concentration gradients of metabolites such as oxygen or glucose (blue line) and waste products such as lactate (red line) within the lower chamber. (B) Images of the top part of the REEC chamber and a (C) higher magnification image of a $200 \mu \mathrm{m}$ diameter opening machined in the glass coverslip. (D) The REEC chamber assembled without the cover on the dish. blocked exchange between cells and bulk media except at the edges, and the cellular environment became hypoxic within tens of minutes (5). This so-called coverslip hypoxia system has been used to model ischemia and reperfusion of cardiomyocytes (22-24). As with spheroids, the sandwich assay is designed to recapitulate an in vivo microenvironment. However, it is not well suited for identifying specific molecules responsible for changes in gene expression or cellular phenotypes that arise as a result. For those types of studies, microfluidic approaches are often needed.

Here we present a new cell culture system, the restricted exchange environment chamber (REEC). REECs build on the principles used in the sandwich system, but they offer several important advantages and new capabilities. REECs can be designed and easily manufactured to produce spatially complex gradients of metabolites in conventional cell culture settings. These cellgenerated gradients affect cell morphology, health, and behavior, and give rise to largescale spatial organization or reorganization of cellular populations as the entire system evolves in time.

\section{REEC Design}

A REEC is a cell culture vessel divided into two parts by a barrier. One portion is a thin planar chamber, and the other is a larger volume holding bulk medium. The planar chamber is defined by a large surface area and a very small height (Figure 1A). The planar chamber is connected to the bulk medium (Figure 1A) via one or more openings in the barrier surface. The size and shape of the chamber and opening(s) are selected such that diffusive exchange between the bulk media and the chamber is restricted and controlled. Cells cultured in the planar chamber produce and consume metabolites, and they generate persistent concentration gradients of metabolites that vary as a function of distance from the opening(s). A single hole design serves as a planar model for a single capillary or blood vessel. However, the number and shape of opening(s) can be designed to model and explore different physiological conditions as well as entirely unnatural situations that may be of interest.

Our development of REECs involved several versions using different types of coverslips, spacers, and clamps (Supplemental Material). In the design presented 
here, a REEC employs a $20 \mathrm{~mm}$ round glass coverslip with one or more small openings machined near the center, and a 100 $\mu \mathrm{m}$ thick O-ring shaped gasket attached to one side near the edge (Figure 1). This coverslip is clamped against the bottom of a glass bottomed dish on which cells have been seeded. The gasket positions the coverslip slightly above the cells and seals the edges, thus forming a shallow sealed chamber that is connected to the bulk medium only through the openings in the coverslip (Figure 1A). When the chamber is formed, cells immediately under the opening continue to experience a bulk environment, but over time, metabolic activity of cells causes gradients to form such that cells further from the opening will exist in lower nutrient and higher waste environments.

\section{Materials and methods \\ REEC fabrication}

REECs were constructed using $20 \mathrm{~mm}$ diameter \#2 glass coverslips and a $35 \mathrm{~mm}$ glass bottom Petri dish (World Precision Instruments, Inc). 100-1000 $\mu \mathrm{m}$ openings in the coverslips were machined manually using a high-speed air drill and tapered carbide bit (SCM Systems, Inc, Menomonee Falls, WI). A $2 \mathrm{~mm}$ wide gasket laser-machined from a 3 mil $(76.2$ $\mu \mathrm{m})$ thick sheet of Mylar was glued to one side of the coverslip using a slow curing 2-part epoxy (Devcon, Danvers, MA). To hold the coverslip in place, a three-armed Mylar clamp was attached to the other side of the coverslip and a $2 \mathrm{~mm}$ wide Mylar ring was glued to the rim of the petri dish. The length of the clamp arms was such that when the coverslip was placed against the bottom of the dish, the arms could be bent slightly and positioned under the rim on the dish, clamping the coverslip against the bottom of the dish (Figure 1B). Note that adhesive adds some thickness to the gasket, there are some edge effects on the Mylar from the laser machining, and there may be some unevenness in the dish that effectively adds height to the chamber. For example, a $76 \mu \mathrm{m}$ thick Mylar gasket affixed to a coverslip using epoxy and placed in a petri dish yielded a final measured chamber height of $\sim 200 \mu \mathrm{m}$, measured by through focusing on an optical microscope. Chamber height uniformity was examined by measuring the height in three chambers at 9-12 uniformly spaced positions. The height variation was $+/-9.4 \%$

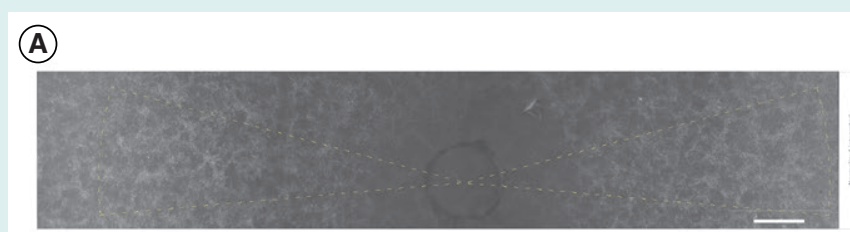

(B)

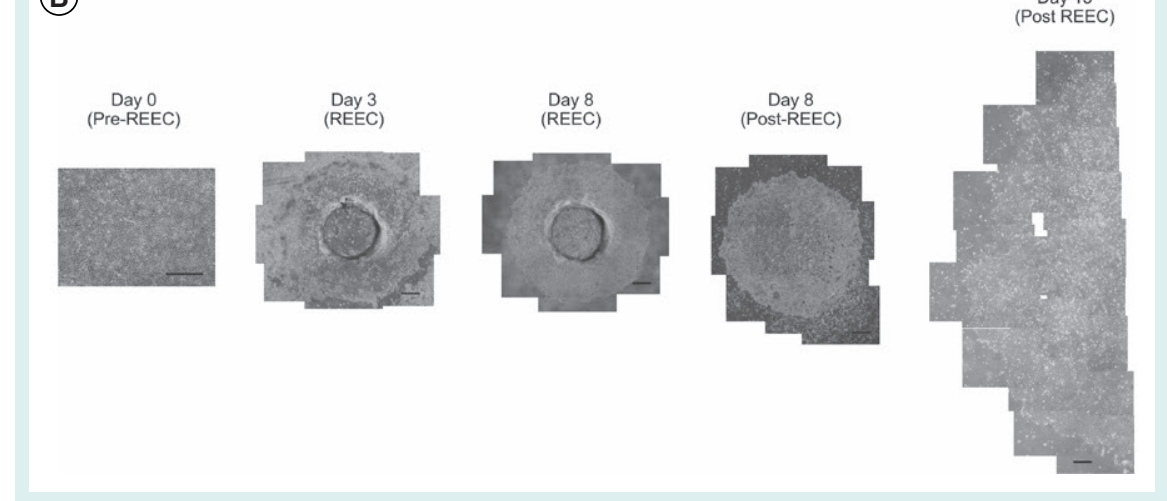

Figure 2. Behavior of cells in a REEC with a single round opening. (A) A hypoxic gradient formed in an REEC. COS-7 cells were treated with the oxygen sensitive dye Image-IT Green and cultured in a REEC for 30 minutes. The probe fluoresces at oxygen concentrations of less than $5 \%$, and the fluorescence intensity increases with decreasing oxygen concentration. Fluorescence imaging shows a radially dependent increase of fluorescence relative to the opening, consistent with a decreasing concentration of oxygen with increasing distance. Dashed lines show the section of the image averaged for the graph. Scale bar, $500 \mu \mathrm{m}$. (B) Time-series experiment using MDCK cells in a REEC. Cells were first plated in a glass bottom dish and grown to confluency. The top half of the REEC chamber was then put in place (Day 0), and the cells returned to the incubator. After three days, cells far from the opening had died, leaving a disk of live cells centered on the opening in the chamber. This disk became more well-defined as the medium exchanges dislodged and rinsed away the dead cells (Day 8). On day 8, the top half of the REEC chamber was removed, exposing the cells to bulk medium and thereby returning them to conventional culture conditions. Cells then began to proliferate, and the disk size increased (e.g. Day 13). Scale bar, $250 \mu \mathrm{m}$.

across the entire chamber, although locally around the opening the variation was likely somewhat less.

\section{Cell culture}

REECs were tested with several different cell lines (ATCC, Manassas, VA), including both epithelial and fibroblast types (see Results), which were maintained in standard cell culture conditions appropriate for each cell type. For experiments in the REECs, cells were plated in a glass bottom dish modified with a Mylar ring on the rim as described above. The dishes were ethanol sterilized for $>15$ min, rinsed well with sterile water, and allowed to dry upside down in a cell culture hood. Cells were seeded in the dishes at a desired density and allowed to adhere for at least several hours. Sometimes the cells were also grown for an extended period time to achieve a desired cell density. Media was then changed to remove unattached cells, and $2 \mathrm{~mL}$ of fresh medium was pipetted into the dish. The top part of the REEC chamber was then put in place. The initial density of cells in an REEC is an important experimental variable, although here we limit the presentation to results for cells plated at near confluency.

For measuring gradients of hypoxia, COS-7 cells were grown to confluency and treated with $2 \mu \mathrm{M}$ Image-IT Green hypoxia reagent (Thermo Fisher Scientific) for 30 min per the manufacturer's protocol. Cells were then rinsed with fresh medium, and the top part of the REEC chamber was put in place. Chambers were held in a tissue culture incubator for 30-60 min and then imaged.

\section{Imaging}

Conventional phase contrast imaging was performed with a Zeiss Axiovert 25 microscope equipped with an eyepiece-mounted CCD camera (AmScope). Antibody labeling and fluorescent staining were performed as previously described (25). Briefly, the REECs were carefully removed from a well, and cells were fixed in paraformaldehyde for $15 \mathrm{~min}$ on ice. Fibronectin was stained with a primary polyclonal rabbit anti-humanFN antibody (Abcam, Cambridge,MA) and then with an AlexaFlour-488-labeled antirabbit secondary antibody from goat (Invitrogen, Carlsbad, CA). Actin was stained 
with phalloidin, and cell nuclei were stained with 4',6-diamidino-2-phenylindole, dihydrochloride (DAPI; Invitrogen). Immunofluorescence microscopy was performed using a Nikon TE200 microscope (Melville, NY), a Coolsnap HQ CCD camera (Roper, Duluth, GA), and Openlab software (Perkin Elmer/ Improvision, Lexington, MA) or an Olympus IX81 with a Hamamatsu C9100-02 EM-CCD camera. FIJI $(\mathrm{NIH})$ was used for image processing and analysis, and the Radial Profile Extended plugin was used to quantify the radial gradients. Fluorescence imaging of the Image-IT Green treated live cells in the REECs was performed using an EVOS FL microscope (Thermo Fisher Scientific) using a 4x objective and standard GFP filter set.

\section{Results and discussion}

The effects of restricted diffusive exchange on cells cultured in REECs were investigated. Several different cell types were successfully cultured in REECs, including MDCK, WI38, COS-7, 3T3, MDA-MB-231, and A549 cells. To demonstrate the presence of a gradient for oxygen, we labeled COS-7 cells with a live cell permeable dye that becomes fluorescent at oxygen levels <5\% (26). Fluorescence imaging after 30-60 min showed a radial gradient, with fluorescence increasing as a function of distance from the opening in the
REEC, indicating that cells farther from the opening experience an increasingly hypoxic environment (Figure 2A).

The long-term behavior of cells in an REEC is illustrated with a time-series experiment using MDCK cells cultured in a REEC with a single round opening. The MDCK cells were plated in a glass bottom dish and grown to confluency (Figure 2B, Day 0). The top of the REEC chamber was put in place, creating a chamber height of approximately $200 \mu \mathrm{m}$, and the dish was placed in a conventional cell culture incubator. The bulk medium was exchanged every two days. No special effort was made to perform the exchange without disturbing the established gradients within the chamber. However, direct oxygen measurements in a similar geometry suggested that the oxygen gradients reform within tens of minutes to an hour (see Supplementary Material). In the example shown, cells distant from the opening began to round up and lose contact with the substrate within less than a day. After three days, there was a well-defined circle of adherent cells that extended about $600 \mu \mathrm{m}$ from the edge of the opening surrounded by what appeared to be dead cells and debris. Media changes required transporting the chamber from incubator to culture hood, and the jostling of the chamber dislodged dead cells and cellular debris within the chamber, some of which were rinsed away through the chamber opening during media changes. After 8 days, a well-defined and radiallysymmetric disk of cells centered on the opening of the REEC could be seen (Figure 2B, Day 8). Notably, the boundary of the disk was sharp, with no individual cells or cells detached from the disk observed. At day 8 , the top of the chamber was removed, releasing the cells from restricted diffusion constraints and exposing them to the bulk medium as in conventional cell culture. Cells at that point began to proliferate (Figure 2B, Day 13) and eventually formed a confluent monolayer across the entire dish.

In addition to the gross radially symmetric morphology that typically develops, different types of radially dependent morphology were observed with different cell types. For example, after extended culture in an REEC, MDCK cells that were beneath the opening exhibited the characteristic cobble stone morphology. In contrast, cells near the edge of the disk had a larger projected area and a 'fried egg' morphology. The human epithelial cell line A549 also formed a contiguous monolayer under the opening. However, in contrast to MDCK cells, the edge of the A549 cell disk was irregular and showed scattered individual cells that had detached from the disk. When fibroblast cell lines (e.g. WI38 and NIH 3T3
A MDCK - under hole

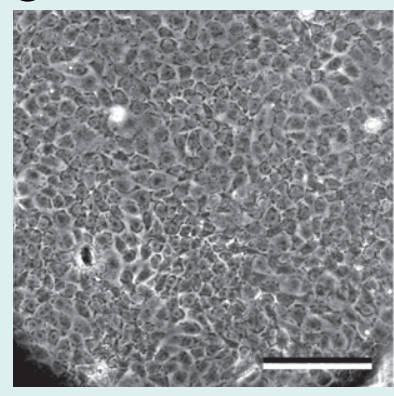

(B) MDCK - edge of cell disk

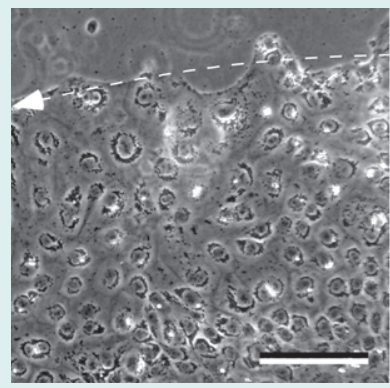

C) A549-under hole

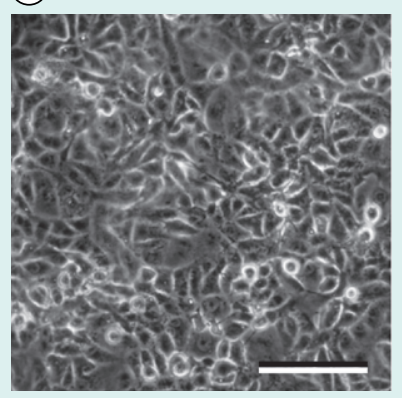

(D) A549 - edge of cell disk

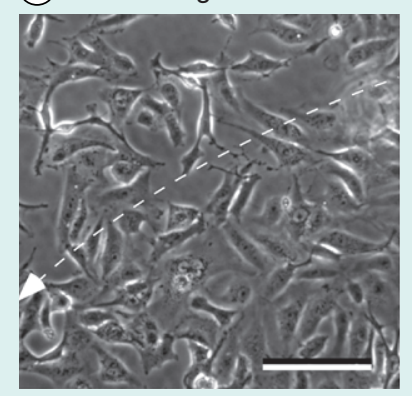

(E) 3Т3 - under hole

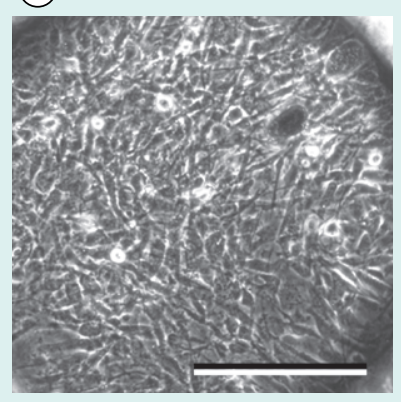

(F) 3T3 - near edge of hole

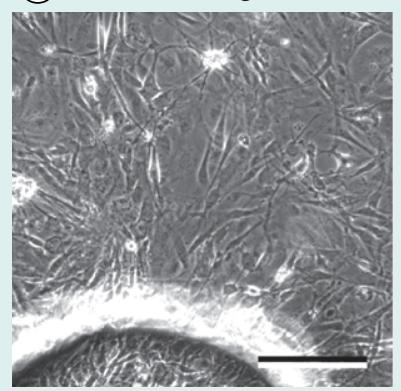

(G) W138-under hole

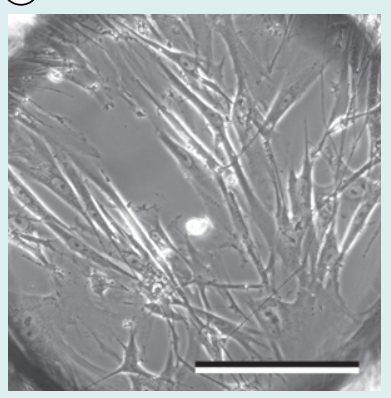

(H) W138 - near edge of hole

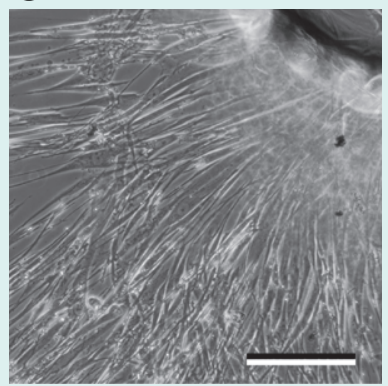

Figure 3. Radially dependent morphologies of cells grown in a REEC. (A) MDCK cells under the opening in contact with the bulk medium. (B) MDCK cells at the edge of the disk of cells. (C) A549 cells under the opening of the REEC. (D) A549 Cells at the edge of the disk of cells. (E) NIH 3 T3 cells under the opening of a REEC. (F) NIH 3 T3 cells extend away from the opening in a REEC. (G) WI38 cells under the opening in a REEC. $(H)$ WI38 cells align radially relative to the opening of a REEC. The light and dark arcs in $(E)$, $(F)$, $(G)$, and $(H)$ are optical scattering effects due to chipping and abrasion on the cover glass on the edge of the microdrilled circular opening. Scale bars, $250 \mu \mathrm{m}$. 
cells) were cultured in REEC chambers, the cells often aligned radially relative to the opening, suggesting that they sense and orient themselves to the diffusive gradients (Figure 3).

One of the advantages of REECs compared to the sandwich system is that openings can be machined in ways that create useful or interesting combinations of gradients. To illustrate the possibilities this offers, we tested a double slot design that is the topological equivalent of a 1D spheroid. In this design the two slots serve as the exterior, and the cell-generated gradients that form between them make the space between the bars the equivalent of the interior of a spheroid (Figure 4A).

MDA-MB-231 cells cultured in a double slot REEC produce a significant amount of extra-cellular matrix (ECM; Figure 4B). Immunofluorescence imaging shows that fibronectin is over-represented in the gap. Also, the fibronectin between the openings is notably anisotropically organized such that the fibers tend to align along the direction of the diffusive gradients, perpendicular to the slots. The fibronectin under the slots has a more isotropic distribution. Cells are present throughout the chamber as reflected by nuclear staining, albeit at a lower density in the gap. There also appears to be a difference in actin expression, with more actin expressed in the cells below the opening.

REECs were developed and designed for modeling tissue-like gradients of metabolites in 2D cell culture. We show that REECs perform as designed for many different types of adherent cells, and a response consistent with an oxygen gradient was demonstrated. Cells grow in REECs in a fashion that is dependent on the distance from an opening in contact with bulk medium. The presumption is that there are gradients of many metabolites extending toward and away from the opening(s). REECs have the same conceptual underpinning as the sandwich assay (11), in which gradients are formed by metabolic activity of cells; however, REECs offer significant advantages.

REECs provide access to new parameters that can be explored using openings of different shapes, sizes, numbers, and organization. One round opening provides a geometry equivalent to a capillary passing through the plane of cells, and a collection of holes provides the equivalent of a capillary bed. We also note that in principle, barriers could be placed in the REEC to impede diffusion, or the liquid medium could readily be replaced with a gel. Variation of the density of cells cultured in the REEC as well as the height of the chamber can alter the gradients formed. The coverslip can also be removed, relieving cells from gradients, analogous to tissue reperfusion experiments (17-20). Furthermore, as with the sandwich assay, REECs offer significant advantages compared to conventional 3D spheroids. They are planar, thus permitting high resolution optical access along the entire axis of diffusion. REECs also do not require cell types that can aggregate into spheroids, however, cells must grow adherently or otherwise be immobilized.

We have demonstrated that cells cultured in REECs elicit multiple phenotypes that are dependent on metabolite gradients and cell type. For example, we find that some types of cells align with the diffusive gradients and deposit ECM along the axis of diffusion. This suggests the possibility that there are cells such as
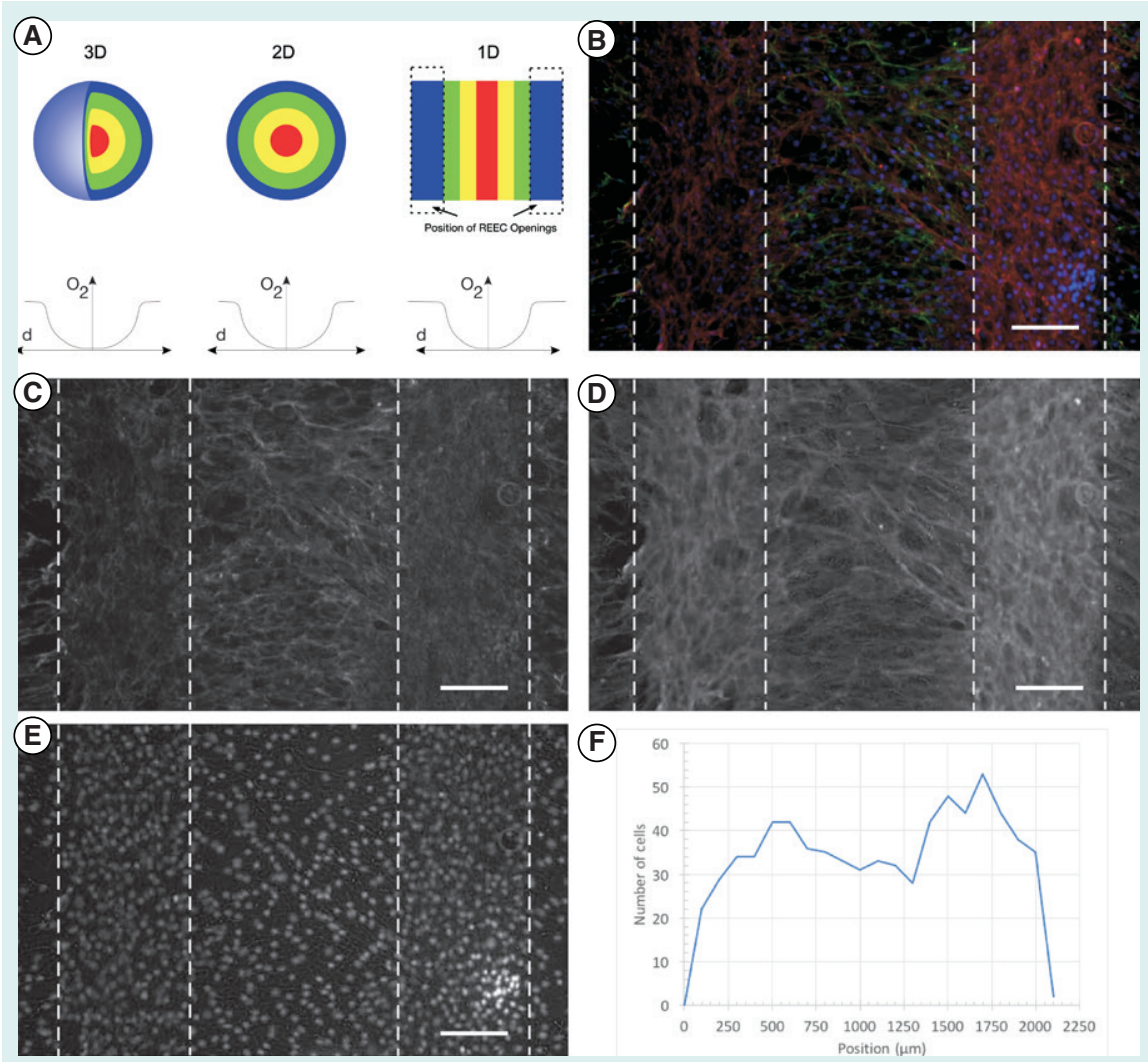

(F)

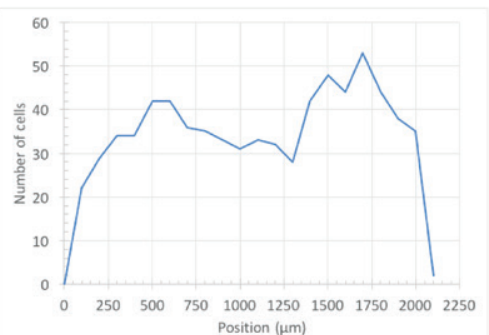

Figure 4. A 1D spheroid model using a REEC. (A) Schematics of a 3D spheroid, an equatorial slice through the 3D spheroid, and 1D spheroid model generated with a REEC. Oxygen concentration as a function of distance is plotted beneath each model. The blue, green, yellow, and red regions represent areas of decreasing oxygen and nutrient concentration. In the 3D spheroid, the red region corresponds to the necrotic core. (B) Fibronectin staining in MDA-MB-231 cells in a REEC with two parallel openings. The openings are shown with the dashed lines. Actin staining (red), nuclei (blue), and fibronectin (green). Individual channels are shown in (C) fibronectin, (D) actin, and (E) nuclei. (F) Cell density as a function of position in the chamber. Scale bars, $250 \mu \mathrm{m}$. 


\section{Acknowledgments}

The authors thank Lew Romer and the JHU School of Medicine Microscope Facility for the use of their microscopes and Julie Dumas, Randy Lawrence, and Caroline Gilmore for technical assistance. We are also grateful to Ross Johnson, Stephen Lockett, and Maria Hoh for helpful discussions. This work was supported in part by a grant from the EU Marie-Curie Program (PIIF-GA-2012-331030).

\section{Competing interests}

The authors declare no competing interests.

\section{References}

1. Egginton, S., and E. Gaffney. 2010. Tissue capillary supply--it's quality not quantity that counts! Exp. Physiol. 95:971-979.

2. Vaupel, P., and M. Hockel. 2000. Blood supply, oxygenation status and metabolic micromilieu of breast cancers: characterization and therapeutic relevance. Int. J. Oncol. 17:869-879.

3. Eales, K.L., K.E.R. Hollinshead, and D.A. Tennant. 2016. Hypoxia and metabolic adaptation of cancer cells. Oncogenesis 5:e190.

4. Walenta, S., S. Snyder, Z.A. Haroon, R.D. Braun, K. Amin, D. Brizel, W. Mueller-Klieser, B. Chance, and M.W. Dewhirst. 2001. Tissue gradients of energy metabolites mirror oxygen tension gradients in a rat mammary carcinoma model. Int. J. Radiat. Oncol. Biol. Phys. 51:840-848.

5. Pitts, K.R., and C.F. Toombs. 2004. Coverslip hypoxia: a novel method for studying cardiac myocyte hypoxia and ischemia in vitro. Am. J. Physiol. Heart Circ. Physiol. 287:H1801-12.

6. Carreau, A., B. El Hafny-Rahbi, A. Matejuk, C. Grillon, and C. Kieda 2011. Why is the partial oxygen pressure of human tissues a crucial parameter? Small molecules and hypoxia. J. Cell. Mol. Med. 15:1239-1253.

7. Jain, M., R. Nilsson, S. Sharma, N. Madhusudhan, T. Kitami, A.L. Souza, R. Kafri, M.W. Kirschner, et al. 2012. Metabolite Profiling Identifies a Key Role for Glycine in Rapid Cancer Cell Proliferation. Science (80-. ). 336:1040-1044.

8. Zigmond, S.H. 1977. Ability of Polymorphonuclear Leukocytes To Orient in Gradients of Chemotactic Factors. J. Cell Biol. 75:606-616.

9. Keenan, T.M., and A. Folch. 2007. Biomolecular gradients in cell culture systems. Lab Chip 8:34-57.

10. Hirschhaeuser, F., H. Menne, C. Dittfeld, J. West, W. Mueller-Klieser, and L.A. Kunz-Schughart. 2010. Multicellular tumor spheroids: An underestimated tool is catching up again. J. Biotechnol. 148:3-15.

11. Fennema, E., N. Rivron, J. Rouwkema, C. van Blitterswijk, and J. De Boer. 2013. Spheroid culture as a tool for creating 3D complex tissues. Trends Biotechnol. 31:108-115.

12. Nath, S., and G.R. Devi. 2016. Three-dimensional culture systems in cancer research: Focus on tumor spheroid model. Pharmacol. Ther. 163:94-108.

13. Pickl, M., and C.H. Ries. 2009. Comparison of 3D and 2D tumor models reveals enhanced HER2 activation in 3D associated with an increased response to trastuzumab. Oncogene 28:461-468.

14. Ingeson-Carlsson, C., A. Martinez-Monleon, and M. Nilsson. 2015. Differential effects of MAPK pathway inhibitors on migration and invasiveness of BRAFV600E mutant thyroid cancer cells in 2D and 3D culture. Exp. Cell Res. 338:127-135.

15. Alemany-Ribes, M., and C.E. Semino. 2014. Bioengineering 3D environments for cancer models. Adv. Drug Deliv. Rev. 79:40-49.

16. Hlatky, L., and E.L. Alpen. 1985. Two-Dimensional Diffusion Limited System For Cell Growth. Cell Prolif. 18:597-611.

17. Hlatky, L., R.K. Sachs, and E.L. Alpen. 1988. Joint oxygen-glucose deprivation as the cause of necrosis in a tumor analog. J. Cell. Physiol. 134:167-178.

18. Hlatky, L., E.L. Alpen, and M.K. Yee. 1986. Differences in the X-Ray Sensitivity of Cells in Different Regions of the Sandwich, a Diffusion-Limited System for Cell Growth. Radiat. Res. 108:62-73.

19. Helmlinger, G., M. Endo, N. Ferrara, L. Hlatky, and R.K. Jain. 2000. Formation of endothelial cell networks. Nature 405:139-141.

20. Carmona-Fontaine, C., V. Bucci, L. Akkari, M. Deforet, J. a Joyce, and J.B. Xavier. 2013. Emergence of spatial structure in the tumor microenvironment due to the Warburg effect. Proc. Natl. Acad. Sci. U. S. A. 110:1940219407.
21. Carmona-Fontaine, C., M. Deforet, L. Akkari, C.B. Thompson, J.A. Joyce, and J.B. Xavier. 2017. Metabolic origins of spatial organization in the tumor microenvironment. Proc. Natl. Acad. Sci. 114:2934-2939.

22. De Diego, C., R.K. Pai, F. Chen, L.H. Xie, J. De Leeuw, J.N. Weiss, and M. Valderrábano. 2008. Electrophysiological consequences of acute regional ischemia/reperfusion in neonatal rat ventricular myocyte monolayers. Circulation 118:2330-2337.

23. Belliard, A., Y. Sottejeau, Q. Duan, J.L. Karabin, and S. V. Pierre. 2012 Modulation of cardiac $\mathrm{Na}+, \mathrm{K}+$-ATPase cell surface abundance by simulated Ischemia/Reperfusion and Ouabain Preconditioning. AJP Hear. Circ. Physiol. 94-103.

24. Solhjoo, S., and B. O'Rourke. 2015. Mitochondrial instability during regional ischemia-reperfusion underlies arrhythmias in monolayers of cardiomyocytes. J. Mol. Cell. Cardiol. 78:90-99.

25. Heinz, W.F., M. Hoh, and J.H. Hoh. 2011. Laser inactivation protein patterning of cell culture microenvironments. Lab Chip. 11:3336-3346.

26. Mandavilli, B.S., A. Chen, and Y. Hu. 2017. Hypoxia measurements in live and fixed cells using fluorescence microscopy and high-content imaging. Mol. Biol. Cell 28:3727 (P3054).

First draft submitted: 4 August 2017; Accepted for publication: 7 February 2018.

Address correspondence to Jan H. Hoh, 6644 Finley Place, Boulder CO 80301, USA, E-mail: jan_hoh@yahoo.com

To purchase reprints of this article, contact: s.cavana@future-science.com

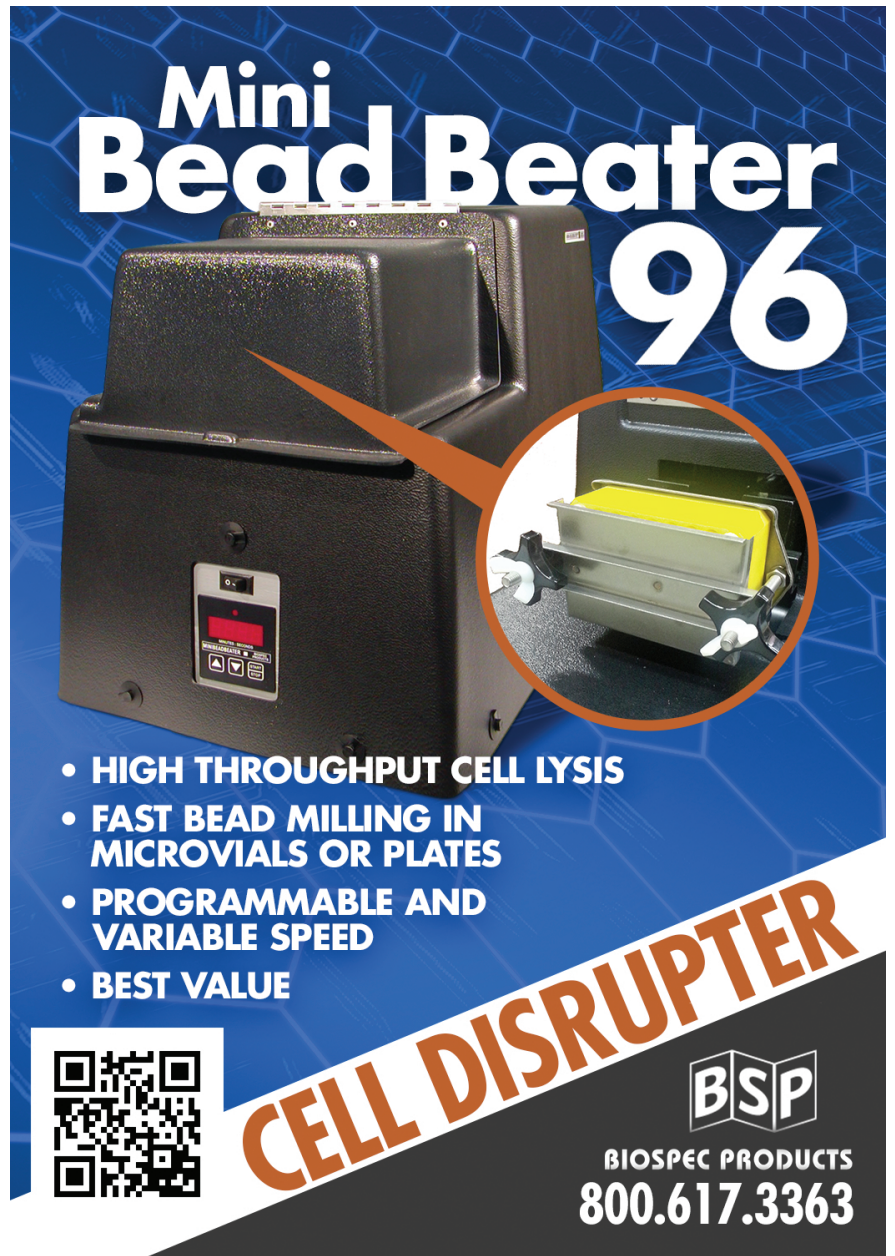

